\title{
A computer-based anaglyphic system for the treatment of amblyopia
}

\section{Ali Rastegarpour}

Ophthalmic Research Center, Shahid Beheshti University of Medical

Sciences, Tehran, Iran
Correspondence: Ali Rastegarpour Ophthalmic Research Center, Shahid Beheshti University of Medical Sciences, 23 Paidarfard St, Boostan $9 \mathrm{St}$. Pasdaran Ave, Tehran 16666, Iran Tel +98 2I 22770957

$\mathrm{Fax}+982122590607$

Email rstgrpr@gmail.com
This article was published in the following Dove Press journal:

Clinical Ophthalmology

15 September 201।

Number of times this article has been viewed

Purpose: Virtual reality (VR)-based treatment has been introduced as a potential option for amblyopia management, presumably without involving the problems of occlusion and penalization, including variable and unsatisfactory outcomes, long duration of treatment, poor compliance, psychological impact, and complications. However, VR-based treatment is costly and not accessible for most children. This paper introduces a method that encompasses the advantages of VR-based treatment at a lower cost.

Methods: The presented system consists of a pair of glasses with two color filters and software for use on a personal computer. The software is designed such that some active graphic components can only be seen by the amblyopic eye and are filtered out for the other eye. Some components would be seen by both to encourage fusion. The result is that the patient must use both eyes, and specifically the amblyopic eye, to play the games.

Results: A prototype of the system, the ABG InSight, was found capable of successfully filtering out elements of a certain color and therefore, could prove to be a viable alternative to VR-based treatment for amblyopia.

Conclusion: The anaglyphic system maintains most of the advantages of VR-based systems, but is less costly and highly accessible. It fulfills the means that VR-based systems are designed to achieve, and warrants further investigation.

Keywords: amblyopia, computer-based, open source, virtual reality, color filters, 3-D

\section{Introduction}

As the development of virtual reality (VR)-based treatment systems such as the Interactive Binocular Treatment (I-BiT ${ }^{\mathrm{TM}}$ ) system presented by Eastgate et $\mathrm{al}^{1}$ and the Viston-VR ${ }^{\mathrm{TM}}$ system presented by Qiu et $\mathrm{al}^{2}$ have demonstrated, the advent of VR technology has been introduced as a promising solution for the management of amblyopia. Preliminary findings imply that VR-based treatment could be effective ${ }^{3}$ and does not involve many of the numerous problems confronted in the conventional approach of occlusion or penalization.

Conventional occlusion therapy, by patching the dominant eye to encourage stimulation of the amblyopic eye, is traditionally the mainstay treatment for amblyopia. ${ }^{4}$ Although effective, ${ }^{5-7}$ this simple intervention produces variable and unsatisfactory outcomes, long durations of treatment, high costs, negative psychological and emotional impacts, and poor compliance, which may even render the treatment completely ineffective. $^{8}$

Atropine penalization of the dominant eye is a recently developed alternative with reportedly better compliance and lower costs, ${ }^{9}$ and of equal efficacy. ${ }^{6,7,9}$ However, 
atropine as a medication has its side effects, ranging from the common and benign experience of light sensitivity, ${ }^{7,10,11}$ to a large variety of less common but more serious symptoms. ${ }^{4}$ Although rare, ${ }^{7,12}$ there have also been reports of reverse amblyopia, ${ }^{13,14}$ a complication in which the unaffected penalized eye becomes amblyopic due to inhibition.

VR-based treatment overcomes many of these problems. VR-based treatment is interactive and adjustable for age and therefore it is enjoyable for the patients and results in excellent patient compliance. ${ }^{1}$ It does not entail the stigmatization of patching or side effects of atropine, and has no risk of reverse amblyopia, since the healthy eye is not occluded or rendered inactive and is not deprived of stimuli. VR-based treatment is said to be successful in preliminary reports. ${ }^{3}$ In addition, while occlusion and penalization can potentially disrupt fusion, VR-based therapy encourages fusion and is expected to enhance binocular vision.

On the other hand, VR-based treatment requires expensive elaborate equipment. It would be costly to implement on a large scale, and it would not be accessible or convenient for most children.

The current paper attempts to introduce a method that could encompass the advantages of VR-based treatment, at a much lower cost. The introduced system can produce an effect similar to the underlying concept of VR-based treatment, using simple technology and obviating the need for complex equipment. The software of this system could be installed on a personal computer at home, and conveniently operated along with a pair of special glasses.

\section{Material and methods}

The essence of VR-based treatment consists of feeding the two eyes two different but related images. Instead of having the two images differ slightly in perspective, as would be intended for three-dimensional (3-D) viewing, the two images would overlap and create a single image, however some elements would be missing for each eye. In particular, there would necessarily be main active elements that would be presented to the amblyopic eye but not to the non-amblyopic eye. Thus, the amblyopic eye would need to play an active role in binocular vision in order to see the complete image, whether it be a video or a game.

VR, however, is not the only method that can be used to feed two different images to the two eyes. Long before the very concept of feeding a different image to each eye was adopted for the treatment of amblyopia, it had been used to create 3-D images and movies. An older technique for creating 3-D experiences was the anaglyphic method. In this method, two images created from a slightly differing point of view were presented in two distinct colors. The viewer would wear a pair of 3-D glasses consisting of two color filters, each to filter one of the images. Therefore, each eye would only see one of the images.

This is the exact mechanism used in the current system. The system consists of a software package and a pair of glasses made of two color filters. The software is designed to be engaging and interactive, but in a manner that at least some of the main active moving components can only be seen by the amblyopic eye and are filtered out for the other eye. This is achieved by simply arranging these elements (and the corresponding backgrounds they cover) to appear in the same colors that the filters allow entry for. Some components, especially the nonmobile or background elements, would be seen by both eyes to encourage fusion. The result is, the patient must use both eyes, and specifically the amblyopic eye, to play the games (Figure 1).

The glasses consisted of two blue (Wratten \#47) and orange (Wratten \#21) generic photographic filters. The filters

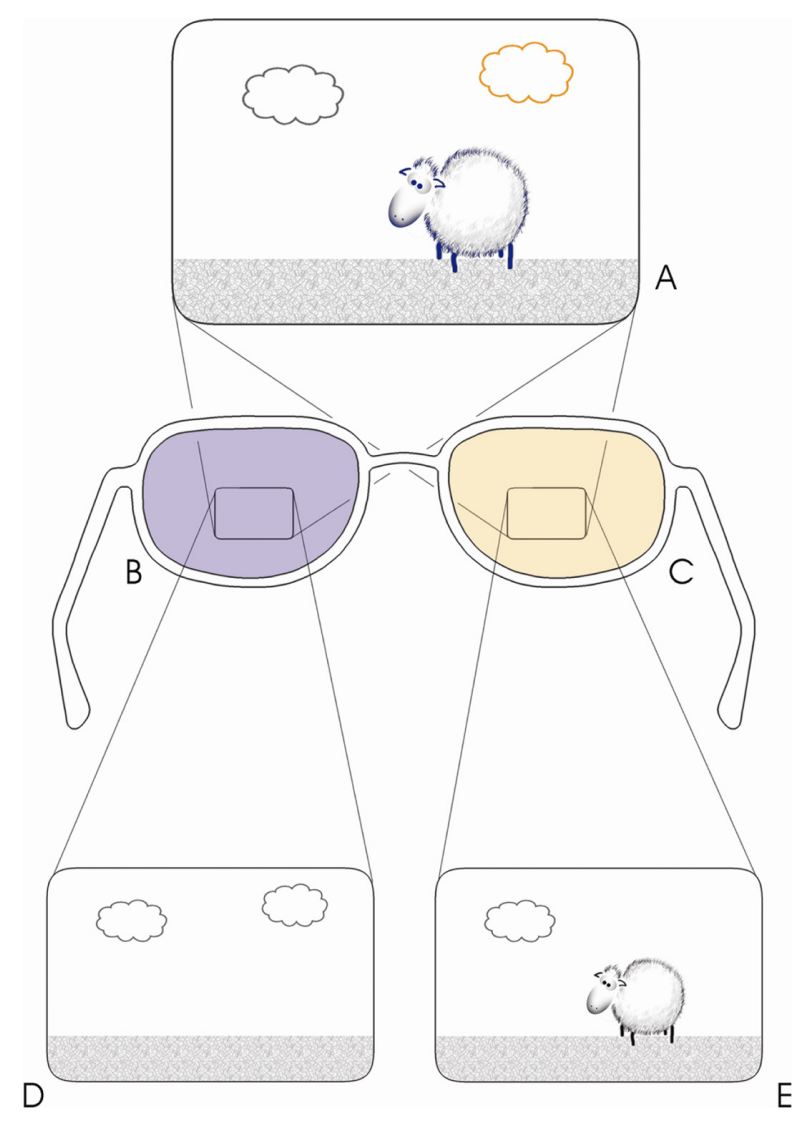

Figure I A diagram of the anaglyphic system for amblyopia treatment. The display (A) consists of elements that, based on color, may be visible by one or both eyes. The filter for the unaffected eye (B) filters out main moving elements (D), while the filter of the amblyopic eye (C) allows for the eye to see the main elements and may or may not filter out less significant features (E). 
were mounted on a frame that adequately covered the field of vision.

Software of the prototype model consisted of simple modified open source Flash (Adobe, San Jose, CA) games. The games used included the open source Flash games of Ping, Xtreme Climber, Snake, and Pacman.

The backgrounds of all games were changed to white, and main elements were changed to the filtered colors. The colors for two different hexadecimal codes were successfully filtered out by each lens. Codes \#99FFFF and \#CCFFFF were filtered by the blue lens and \#FFFF33 and \#FFFF99 were filtered by the orange lens. This enabled us to create images with three shades (two shades of color and white), which could be filtered out for one eye.

\section{Results}

A laboratory prototype of the proposed system, the ABG InSight (v1.2 $\beta$ ), was designed. The system was used on nine monitors, with different manufacturers and models, and complete filtering was confirmed by twelve people without a history of any ophthalmologic or neurological problems. A simple calibration module could be added to the software later to guarantee consistency in filtering elements, or for the time being the monitors could be adjusted by any person without color vision deficits, to ensure correct filtering.

The glasses consisted of two generic photographic filters, which were for the purpose of this study, blue and orange, but other color pairs, such as the traditional 3-D red-cyan or amber-pure dark blue (used in ColorCode 3-D) ${ }^{15}$ would presumably be equally functional.

The prototype system was capable of successfully filtering out elements of a certain color and therefore, was found to be a potential alternative to VR for amblyopia management.

\section{Discussion}

As mentioned, the computer-based anaglyphic system provides most of the advantages of the VR-based treatment, in addition to reduced cost and high availability.

The open source initiative allows for the modification, and in most cases, distribution, of a variety of software packages, free of charge and licensing. This creates the opportunity for researchers to gain access to libraries of software, and from the many available programs, select and use those that may suit their purpose. In this case, applying a few simple changes in the code of a game, such as changing the color of the elements, could make it completely compatible with the proposed system. For this means, many of the available games can be used, taking into consideration only the appropriateness of the game for the target age group, and complexity of the graphic interface. The license of some open source games does not allow them to be modified for commercial use. This should be taken into consideration, the license respected, and no financial gain received from such games.

One of the limitations for such a system would be the main limitation of all anaglyphic systems: the limited use of color. Games that include color as a main theme or include color-based elements, as well as games and media with complex graphics, would be slightly restricted. Although anaglyphs can reproduce color images and to a point, color distinction and clarity, the scope of options is limited. For example, the main moving elements, as well as other components which are selected for filtering, along with the corresponding backgrounds they cover, must be invariably monochrome. For this reason, the background and main elements can only consist of white and various shades of the filtered color. In most cases, between the darkest shade of the filtered color and white, only one distinctively visible shade will be practical for use. This limits the colors for use in the main elements and backgrounds to three colors; white and the two shades of the filtered color. Aside from this issue the use of various points of view, perspectives, and movements remain unrestricted.

A minor advantage for some VR-based systems would be that they can be made to adjust for angles of strabismus, which means they can be used for untreated strabismic amblyopes and adjusted as such to provide binocular vision and fusion without requiring satisfactory alignment. Since the anaglyphic system uses a single display, its use is limited to amblyopic patients for whom the underlying condition, usually strabismus or anisometropia, has been resolved, at least to some extent, by corrective glasses or other means.

A minor advantage of the anaglyphic system is that the fusion promoted for seeing the images in this system, is similar to the fusion required in the actual world, because both eyes are watching the same interface. VR-based systems may not represent the actual angles, distances, or proportions seen in the natural surroundings. This is why prolonged work with VR systems has been associated with vomiting, sweating, headaches, and drowsiness. ${ }^{16}$

The anaglyphic system has much potential to become a large-scale open source research project. Various open source applications could be modified by volunteers to enrich the library of software used in the project, and researchers throughout the world could use standard filters to create the glasses, and download the software free of charge. 
A major concern is the actual effectiveness of the VR-based systems. Although the anaglyphic system could potentially serve as an alternative to VR-based systems by accomplishing the same objectives, the evidence supporting VR-based systems as a therapeutic intervention is limited, and the only available studies including clinical data in this regard are two case series reporting the short-term outcomes in six and twelve patients, respectively. ${ }^{3,17}$ Computer-based active vision therapy has received much attention for amblyopia and one of the recent publications by Hess et al, ${ }^{18}$ demonstrating success for active vision therapy in three amblyopic patients. However, there is still not much evidence in the literature to support most modalities. Nonetheless, introducing the anaglyphic system provides an excellent opportunity to investigate the role of computer-based therapy in the management of amblyopia, by enabling researchers worldwide to evaluate its effectiveness without the need for expensive or exclusive equipment, and therefore allowing interested researchers to continue from where the previous studies were left.

\section{Conclusion}

The current lack of clinical data for the amblyopic system is a major drawback of this introductory paper. However, it has not been claimed that the anaglyphic method is an effective treatment for amblyopia, but rather that the system could logically be a suitable alternative to the VR systems. The cost of anaglyphic systems is much lower, therefore they may be a more viable option for research and maybe ultimately, treatment. The evidence for VR-based systems could be intriguing enough for researchers to test an anaglyphic system that functions similarly, with better availability and lower costs.

Future clinical trials performed on VR-based treatment systems can document the effectiveness of the underlying concept, on which the current system was designed. In addition, clinical trials and case series performed with the anaglyphic system itself will determine its true effectiveness and implications.

In conclusion, the anaglyphic system maintains most advantages of the VR-based systems, but is less costly and more accessible. The system logically fulfills what the VR-based system was designed to achieve and therefore, warrants further investigation.

\section{Acknowledgments}

The author would like to express gratitude to the following persons who had an indispensable role in the present study:
Dr Zhaleh Rajavi at the Ophthalmic Research Center of Shahid Beheshti University of Medical Sciences; Behzad Daemi and Dr Alireza Asary Yazdi for their help and advice regarding software and gaming; and Dr Alireza Namazi at Tehran University of Medical Sciences. The "Ping" source code was created and released by Jim Bumgardner, "Xtreme Climber" by Matthew Strange, and "Snake Game" by Mahesh Kokadwar.

\section{Disclosure}

The author is the patent holder of the system described in the manuscript protected by Patent No. 64330; 89/01/31 at the Iran Patent Office; however this does not translate to any personal commercial gain. The author has not been the recipient of any monetary profit from the device, and does not intend to be in the future.

\section{References}

1. Eastgate RM, Griffiths GD, Waddingham PE, et al. Modified virtual reality technology for treatment of amblyopia. Eye (Lond). 2006;20(3): 370-374.

2. Qiu F, Wang L, Liu Y, Yu L. Interactive binocular amblyopia treatment system with full-field vision based on virtual reality. The 1st International Conference on Bioinformatics and Biomedical Engineering 2007 (ICBBE 2007) July 6-8, 2007; Wuhan, China: Institute of Electrical and Electronics Engineers (IEEE); 2007:1257-1260.

3. Waddingham PE, Butler TK, Cobb SV, et al. Preliminary results from the use of the novel Interactive binocular treatment (I-BiT) system, in the treatment of strabismic and anisometropic amblyopia. Eye (Lond). 2006;20(3):375-378.

4. Webber AL. Amblyopia treatment: an evidence-based approach to maximising treatment outcome. Clin Exp Optom. 2007;90(4): 250-257.

5. Teed RG, Bui CM, Morrison DG, Estes RL, Donahue SP. Amblyopia therapy in children identified by photoscreening. Ophthalmology. 2010; 117(1):159-162.

6. Repka MX, Kraker RT, Beck RW, et al. Pediatric Eye Disease Investigator Group. A randomized trial of atropine vs patching for treatment of moderate amblyopia: follow-up at age 10 years. Arch Ophthalmol. 2008;126(8):1039-1044.

7. Scheiman MM, Hertle RW, Kraker RT, et al. Pediatric Eye Disease Investigator Group. Patching vs atropine to treat amblyopia in children aged 7 to 12 years: a randomized trial. Arch Ophthalmol. 2008; 126(12):1634-1642.

8. Awan M, Proudlock FA, Grosvenor D, Choudhuri I, Sarvanananthan N, Gottlob I. An audit of the outcome of amblyopia treatment: a retrospective analysis of 322 children. Br J Ophthalmol. 2010;94(8):1007-1011.

9. Li T, Shotton K. Conventional occlusion versus pharmacologic penalization for amblyopia. Cochrane Database Syst Rev. 2009;4: CD006460.

10. Pediatric Eye Disease Investigator Group. Pharmacological plus optical penalization treatment for amblyopia: results of a randomized trial. Arch Ophthalmol. 2009;127(1):22-30.

11. Repka MX, Kraker RT, Beck RW, et al. Pediatric Eye Disease Investigator Group. Treatment of severe amblyopia with weekend atropine: results from 2 randomized clinical trials. J AAPOS. 2009; 13(3):258-263.

12. North RV, Kelly ME. Atropine occlusion in the treatment of strabismic amblyopia and its effect upon the non-amblyopic eye. Ophthalmic Physiol Opt. 1991;11(2):113-117. 
13. Kubota N, Usui C. The development of occlusion amblyopia following atropine therapy for strabismic amblyopia. Nippon Ganka Gakkai Zasshi. 1993;97(6):763-768. Japanese.

14. Simons K, Stein L, Sener EC, Vitale S, Guyton DL. Full-time atropine, intermittent atropine, and optical penalization and binocular outcome in treatment of strabismic amblyopia. Ophthalmology. 1997;104(12): 2143-2155.

15. Sorensen SEB, Hansen PS, Sorensen NL, inventors. Method for recording and viewing stereoscopic images in color using multichrome filters. United States Patent 6687003. May 31, 2001.
16. Oman CM. Sensory conflict in motion sickness: an observer theory approach. In: Ellis SR, editor. Pictorial Communication in Virtual and Real Environments. London, UK: Taylor and Francis; 1993:362-376.

17. Cleary M, Moody AD, Buchanan A, Stewart H, Dutton GN. Assessment of a computer-based treatment for older amblyopes: the Glasgow Pilot Study. Eye (Lond). 2009;23(1):124-131.

18. Hess RF, Mansouri B, Thompson B. A binocular approach to treating amblyopia: antisuppression therapy. Optom Vis Sci. 2010;87(9): 697-704.
Clinical Ophthalmology

\section{Publish your work in this journal}

Clinical Ophthalmology is an international, peer-reviewed journal covering all subspecialties within ophthalmology. Key topics include: Optometry; Visual science; Pharmacology and drug therapy in eye diseases; Basic Sciences; Primary and Secondary eye care; Patien Safety and Quality of Care Improvements. This journal is indexed on

Submit your manuscript here: http://www.dovepress.com/clinical-ophthalmology-journal

\section{Dovepress}

PubMed Central and CAS, and is the official journal of The Society of Clinical Ophthalmology (SCO). The manuscript management system is completely online and includes a very quick and fair peer-review system, which is all easy to use. Visit http://www.dovepress.com/ testimonials.php to read real quotes from published authors. 